\title{
La genética del trastorno antisocial de la personalidad: Una revisión de la bibliografía
}

\author{
Tomás Efrén Holguín Mendoza, ${ }^{1}$ Juan Jorge Palacios Casados²
}

Actualización por temas

\section{SUMMARY}

The antisocial personality disorder (ASPD) is characterized by an incapacity for an individual to adapt himself or herself to the social norms. These social norms are extremely important because they govern many aspects of behavior during adolescence and adulthood. Patients with ASPD typically have irritability problems and aggressive feelings toward other people. These aggressive feelings toward other people are expressed in a context of threat and intimidation. The ASPD is less common in clinical settings comparing the total population, in which the prevalence is 1.1. The familial aggregation for ASPD has been registered, in which the $40-50 \%$ of the variance can be explained to genetic influences. Most of the studies of ASPD in molecular genetics have been applying the hypothesis of association of candidate genes, focusing on genes associated with neurotransmission pathways. This has been greatly relevant to the monoamine oxidase gene (MAO). Genes that promote specific behavior between individuals must have been selected through the process of natural selection. Aggressive behaviors and other types of behavior that have an evolutionary origin are similar in the fact that they have to be codified in the genes and will later be transmitted to their descendants.

Key words: Antisocial personality disorder, cuantitative genetics, molecular genetics.

\section{RESUMEN}

El trastorno antisocial de la personalidad (TAP) consiste en una incapacidad para adaptarse a las normas sociales que habitualmente rigen numerosos aspectos de la conducta de las personas en la adolescencia y la edad adulta. Los pacientes con TAP característicamente tienen problemas de irritabilidad y sentimientos agresivos hacia los demás, los cuales se expresan en el contexto de la amenaza o la intimidación. El TAP es menos común en la clínica comparándolo con la población general en la que se reporta una prevalencia media del 1.1. Se ha registrado una agregación familiar para el TAP en la que el $40-50 \%$ de la varianza puede ser explicada por influencias genéticas. La mayoría de los estudios de genética molecular en el TAP se han realizado utilizando la hipótesis basada en los estudios de asociación con genes candidatos, enfocándose en los genes relacionados a vías de neurotransmisión, siendo uno de los más relevantes, hasta el momento, el gen para la monoamino oxidasa (MAOA). Aquellos genes que promueven que cierta conducta exista entre los individuos debieron haberse elegido a través del proceso de la selección natural. De manera similar a otros comportamientos que tienen orígenes evolutivos, los comportamientos agresivos también deben ser codificados en los genes, que a la postre serán transmitidos a la descendencia.

Palabras clave: Trastorno antisocial de la personalidad, genética cuantitativa, genética molecular.

\section{DEFINICIONES}

Un trastorno de la personalidad (TP) es definido por el DSM-IV-TR (Diagnostic and Statistical Manual of Mental Disorders) como un patrón rígido de experiencia interna y de conducta que se desvía marcadamente de las expectativas culturales del individuo, es persistente e inflexible, tiene su inicio en la adolescencia o en la adultez temprana, es estable a través del tiempo y conlleva malestar o deterioro. ${ }^{1}$

Uno de los aspectos más antiguos y controversiales en el campo de la clasificación de los TP ha sido si éstos debe- rían ser conceptualizados dimensional o categóricamente. Parece ser que existe un acuerdo general de que los TP se clasificarían mejor de manera dimensional, ${ }^{2-4}$ y se discutieron distintos sistemas alternativos para el DSM-5. ${ }^{5}$

Sólo los criterios del trastorno antisocial de la personalidad (TAP), de entre el resto de los trastornos de personalidad, han producido históricamente niveles aceptables de confiabilidad, y esos criterios han enfatizado los actos abiertamente criminales o delictivos.

El TAP consiste en una incapacidad para adaptarse a las normas sociales que habitualmente rigen numerosos as-

Hospital de Especialidades Dr. Bernardo Sepúlveda. Centro Médico Nacional Siglo XXI. Instituto Mexicano del Seguro Social.

2 Clínica de Genética Psiquiátrica. Instituto Nacional de Psiquiatría Ramón de la Fuente Muñiz.

Correspondencia: Dr. Tomás Efrén Holguín Mendoza. Calle Dr. Velasco 14 int. 51 1B, Doctores, Cuauhtémoc, 06720. México, D.F. Teléfono: (55) 5588-9369.

Celular: (55) 4487-2812. E-mail: praximeo@me.com

Recibido: 27 de febrero de 2013. Aceptado: 30 de octubre de 2013. 
pectos de la conducta de las personas en la adolescencia y la edad adulta. Los pacientes con TAP característicamente muestran fácilmente irritabilidad y sentimientos agresivos hacia los demás, los cuales se expresan en el contexto de la amenaza o la intimidación.

El DSM-IV-TR lo describe como un trastorno caracterizado por un patrón general de desprecio y violación de los derechos de los demás que se presenta desde la edad de 15 años.

La investigación longitudinal ha documentado que los niños y los adolescentes con trastornos de la conducta tienen un mayor riesgo para presentar conductas antisociales en la adultez. ${ }^{6}$

Aunque no todas las conductas antisociales de la adultez tienen antecedentes en la infancia, ${ }^{7}$ los trastornos con factores genéticos contribuidores tienden a manifestarse de forma temprana en la vida. Hasta la mitad de aquellos que presentan un trastorno de conducta de manera temprana pueden aún experimentarlo en la adultez, mientras que los individuos con inicio más tardío de los síntomas pueden experimentar síntomas transitorios y lograr la remisión. ${ }^{8-11}$

\section{EPIDEMIOLOGÍA}

En 12 estudios de población general que analizaron la prevalencia de distintos trastornos de personalidad, reportaron prevalencias para el TAP que iban del 0.2 al 4.5, con una media de 1.1. El primer estudio reportado es de 1989 y el último, del 2007. Fueron realizados en los EUA, el Reino Unido y Noruega, con entrevistas estructuradas del DSM-III, DSM-III-R y DSM-IV como instrumentos de medición según cada época. ${ }^{12}$

El TAP es menos común en la clínica comparándolo con la población general. Esto se debe a que los pacientes con patrones de pensamiento y conducta antisocial rara vez buscan tratamiento. ${ }^{13}$

Se presenta con mayor frecuencia en hombres que en mujeres. ${ }^{14-16}$ Dentro del cluster B de los trastornos de personalidad del DSM-IV-TR, las personas con rasgos o franco TAP suelen ser mas jóvenes, ${ }^{17}$ con mayor frecuencia están divorciadas, separadas o nunca se han casado; cuando son entrevistadas frecuentemente están solteras y viven solas.

Algunos estudios documentan que el TAP -o personas con estos rasgos-, se encuentra relacionado con una baja educación. Un estudio del 2006 encontró que todos los trastornos del cluster B estaban asociados a una clase social baja; ${ }^{18}$ y en un estudio del 2004 se documentó una asociación con ingresos menores; ${ }^{19}$ pero un estudio del 2002 no había encontrado ninguna asociación con el ingreso. ${ }^{20}$

\section{Datos en México}

En las estadísticas sobre menores infractores se puede observar un incremento en el número de los sujetos puestos a disposición del Consejo de Menores, es decir, de aquellos sometidos a algún proceso penal; de 2623 registrados en el año 1999, la cifra se elevó a 3506 en el 2003. ${ }^{19,21}$ Se ha observado que es mayor la proporción de hombres que cometen infracciones y, además, que los adolescentes son puestos a disposición del Consejo de Menores a una edad más temprana. Los robos constituyen la principal infracción, pero en años más recientes se documentan infracciones que, aunque cometidas por una escasa proporción de adolescentes, se pueden considerar de mayor gravedad, por ejemplo el homicidio, la portación de armas prohibidas, la violación y el abuso sexual. ${ }^{22}$

Al revisar la conducta antisocial en los estudiantes adolescentes de México, los resultados más importantes indican también que es mayor la proporción de los hombres que incurren en este tipo de comportamientos, y que destacan la participación en riñas, el golpear o dañar cosas ajenas, el golpear o herir a otras personas y tomar dinero ajeno. Los comportamientos considerados de mayor gravedad como vender drogas y usar un arma para robar o atacar a alguien, se han documentado en una proporción baja de estudiantes. $^{23-26}$

En mediciones que se realizaron en 1997, 2000 y 2003, en estudiantes adolescentes del nivel escolar medio y medio superior de escuelas públicas y privadas del Distrito Federal, en un estudio que buscaba las tendencias de la conducta antisocial, se encontró que de 1997 al 2000 hubo un incremento del 1.9\% de cualquiera de las conductas antisociales, y del 2000 al 2003 el aumento fue de 6.7\%. En términos generales, el estudio concluye que hubo un incremento para conductas antisociales no graves relacionadas con violencia y robo, como lo serían tomar parte en riñas, golpear o danar objetos, golpear o herir a personas, tomar mercancía sin pagarla y robar dinero o valores por 50 pesos o menos; así como de conductas antisociales graves, específicamente la sustracción de dinero o valores mayores a 500 pesos y atacar a alguien usando un objeto o arma, aunque esta última conducta con incrementos menores. ${ }^{27}$

Estas situaciones se presentaron de manera similar en los hombres y en las mujeres, aunque éstas aún representan una proporción menor, con una relación de casi dos hombres por cada mujer.

En el presente texto nos enfocaremos en los factores genéticos del TAP, haciendo referencia a la interacción que parece darse entre genes y ambiente para que se desarrolle el trastorno o algún rasgo, a la vez que se considerará una perspectiva evolutiva de estas conductas.

\section{ASPECTOS GENÉTICOS}

La genética psiquiátrica es un campo multidisciplinario con raíces en la genética humana, la psiquiatría, las estadísticas y la epidemiología, que data de poco más de 100 años. El trabajo más temprano en este campo involucró métodos de genética 
La genética del trastorno antisocial de la personalidad

clínica y conductual (o genética cuantitativa), como estudios de familia, de gemelos y de adopción, los cuales son efectivos para establecer si los factores genéticos influyen en un rasgo y en qué grado. Subsecuentemente, el campo se diversificó para incluir métodos de genética molecular como los análisis de ligamiento y de asociación, que se suponía permitirían el aislamiento de regiones de cromosomas (locus candidatos) y la identificación de genes específicos que estuvieran mediando la transmisión familiar de un rasgo conductual.

\section{Genética cuantitativa}

Los rasgos normales de personalidad han mostrado repetidamente estar influidos por factores genéticos, con estimaciones que van de un $30 \%$ a un $60 \%$ de heredabilidad aproximadamente. ${ }^{28,29}$ Estimaciones similares de heredabilidad han sido encontradas en clasificaciones dimensionales de los TP basadas en autorregistros. ${ }^{30}$

Los estudios de epidemiología genética indican que los 10 trastornos de personalidad clasificados en el eje II del DSM-IV-TR son de leve a moderadamente heredables.

\section{Estudios de familia}

Se ha registrado agregación familiar para el TAP. ${ }^{31}$ Existe un patrón familiar que se manifiesta en una frecuencia cinco veces mayor entre los familiares de primer grado de los varones afectados que entre los familiares de los individuos de control. ${ }^{32}$

El TAP parece tener tanto raíces genéticas como ambientales. Un adulto adoptado que tiene un padre biológico con registros de haber estado en prisión por haber mostrado conductas antisociales tiene cuatro veces más probabilidades de presentar una problemática conducta agresiva que una persona sin tal vulnerabilidad biológica. Al mismo tiempo, una persona en la que su padre adoptivo tiene un TAP, tiene más de tres veces la probabilidad para desarrollar el trastorno (comparado con la población general sin este antecedente), a pesar de la historia biológica. ${ }^{33}$

\section{Estudios de gemelos y adopción}

En un estudio de población con gemelos utilizando representaciones dimensionales para los TP del cluster B del DSM-IV, se encontró una heredabilidad para el TAP del 38\%. ${ }^{34}$ Los factores genéticos aditivos dan razón del 49\% de la varianza y son más importantes que el ambiente compartido para la psicopatología. ${ }^{35}$

En su texto Psychiatric Genetics: A Primer (2008), Tsuang estima que la heredabilidad (h2) para el TAP es del 69\%, equivalente al trastorno límite de la personalidad, encontrándose por debajo del tartamudeo y por encima del trastorno obsesivo compulsivo. ${ }^{36}$
Una revisión meta-analítica del TAP, de 1997, sugiere que el $40-50 \%$ de la varianza puede ser explicada por influencias genéticas. ${ }^{37}$ A su vez, en un meta-análisis realizado por Rhee et al. en 2002, con 51 estudios de gemelos y de adopción de conductas antisociales basado en registros, autorreportes y reportes familiares, se encontró que la varianza podría ser explicada por factores genéticos aditivos (32\%), factores genéticos no aditivos (9\%), factores por ambiente compartido (16\%) y factores de ambiente individual específico $(43 \%),{ }^{35}$ sin existir diferencias significativas en la magnitud de las influencias genéticas y ambientales para hombres y mujeres. En un meta-análisis más reciente, Ferguson encontró en el 2010 que las influencias genéticas representaron el componente más importante en la varianza del TAP, con un 56\%; los factores por ambiente compartido con un $11 \%$ y los factores de ambiente individual especifico con un $31 \%$. Al mismo tiempo se encontró que las influencias genéticas tenían un mayor poder predictivo para el TAP en los individuos jóvenes que en los adultos. Estos resultados indican que el componente genético es un contribuyente importante para el TAP pero que las influencias no genéticas, particularmente las experiencias de vida únicas, también son importantes. ${ }^{38}$

Sea como sea, de acuerdo a la información que se tiene para el TAP y otros trastornos de la personalidad, es importante recordar que todas las estimaciones de heredabilidad son dependientes de la muestra.

Un estudio de Torgersen et al. (2008) en una población de gemelos, que incluía todos los TP del cluster $\mathrm{B}$, indicó que el TAP parecía contener muchos más factores de riesgo genético que aquellos contenidos en común con otros TP del cluster B, a excepción del trastorno límite de la personalidad.

Numerosos estudios de familia, de gemelos y de adopción han demostrado que el TAP, los trastornos de la conducta y los trastornos por consumo de sustancias (frecuentemente denominados trastornos externalizados), conllevan un riesgo genético común..$^{39,40}$ En un estudio de familias con gemelos realizado por Hicks et al. (2004), se encontró una alta vulnerabilidad general heredable $(80 \%)$ para todos los trastornos externalizados, que representó a la mayoría de los parentescos familiares. ${ }^{41}$

En un estudio de gemelos realizado por Lyons et al. (1995), se demostró que la influencia genética en los síntomas del TAP del DSM-III-R era más prominente en los adultos que en los adolescentes. ${ }^{42}$

Todos estos resultados sugieren que las influencias genéticas tienen una contribución significativa para el TAP.

En 1974, Crowe ${ }^{43}$ encontró que el cuidado institucional temprano era un factor de riesgo para la posterior conducta antisocial sólo cuando se encontraba presente un factor de riesgo genético. En otro estudio de adopción, Cadoret et al. $(1983)^{44}$ encontraron una significativa interacción gen-ambiente cuando mostraron que existía un riesgo despreciable para la conducta antisocial si sólo se tenía el factor de ries- 
go genético (conducta antisocial en el padre biológico) y un nulo efecto si sólo existía un ambiente adverso en la familia adoptiva, pero que existía un efecto sustancial cuando ambos factores de riesgo estaban presentes. Este hallazgo fue replicado después en un estudio con un mayor número de adoptados. ${ }^{45}$ Jaffe et al. (2005), ${ }^{46}$ utilizando un diseño de gemelos, encontraron una interacción significativa gen-ambiente con respecto al maltrato infantil y el desarrollo de la conducta antisocial, y en un estudio de gemelos Tuvblad et al. (2006) ${ }^{47}$ demostraron una significativa interacción genambiente al demostrar que la heredabilidad para la conducta antisocial en adolescentes es mayor en entornos socioeconómicos desfavorecidos. Al utilizar un avanzado estudio de familia, Feinberg et al. (2007) ${ }^{48}$ encontraron una interacción del genotipo con la negligencia parental, así como con el escaso afecto, prediciendo la conducta antisocial. En un estudio de población con gemelos, Hicks et al. (2009) demostraron una significativa interacción gen-ambiente con distintos factores de riesgo ambientales, mostrando que una mayor adversidad ambiental se asociaba con un riesgo genético incrementado para el TAP y el trastornos por consumo de sustancias. ${ }^{49}$

\section{Genética molecular}

La mayoría de los estudios de genética molecular en el TP se han realizado utilizando la hipótesis basada en los estudios de asociación con genes candidatos, enfocándose en los genes relacionados a vías de neurotransmisión, especialmente en los sistemas serotoninérgico y dopaminérgico. Ejemplos de genes relacionados al metabolismo incluyen a la cateco-Ometil transferasa (COMT), la monoamino oxidasa (MAOA) y la dopamina beta-hidroxilasa $(\mathrm{DBH})$; aquellos relacionados a la morfología del receptor incluyen al receptor D2 de dopamina (DRD2), al receptor D4 de dopamina (DRD4), al receptor $1 \mathrm{~B}$ de serotonina (5HTR1B) y al receptor 2A de serotonina (5HTR2A); y aquellos relacionados a la actividad de transportadores incluyen al transportador de serotonina (en particular un polimorfismo en la región promotora del gen referido como "transportador de serotonina ligado a las regiones polimórficas" o 5HTTLPR) y el transportador de dopamina (DAT).

\section{Análisis de ligamiento y de asociación}

Múltiples líneas de evidencia sugieren que una disfunción en el sistema de la serotonina (5-HT) está asociada con la impulsividad, la agresión, la labilidad afectiva y el suicidio. Los genes ligados a la función de estos neurotransmisores pueden ser considerados, por lo tanto, genes candidatos para el TAP.

Localizado en el cromosoma 17 (17q11.2), el transportador de serotonina (5HTT o SLC6A4) codifica una proteína transportadora que remueve la serotonina del espacio sináp- tico y la introduce a las neuronas presinápticas. Este sitio es un blanco de acción de los medicamentos antidepresivos. Un repetido en tándem de número variable (del inglés VNTR -variable number tandem repeat) en el promotor de este gen (5HTTLPR) ha mostrado que afecta la proporción de recaptura de serotonina y puede desempeñar un papel en la conducta enferma, con los alelos cortos en este polimorfismo esencialmente dialélico (ya sea corto o largo) siendo relacionados con niveles bajos de transcripción de este gen. ${ }^{44}$

Cadoret et al. (2003) encontraron una relación entre la baja actividad de la variante corta del 5HTTLPR y el espectro de los trastornos externalizados ante la presencia de adversidad ambiental después del control de otras variables, tales como el sexo y la edad. ${ }^{50}$

Un estudio de Lyons-Ruth et al. (2007) encontró una relación significativa entre el alelo corto de 5HTTLPR y el TA, ${ }^{51}$ pero otros estudios han fallado en encontrar una asociación entre ese polimorfismo y algún trastorno de personalidad del cluster B. ${ }^{52}$

En un estudio de Retz et al. (2004) se encontró una relación entre el gen promotor del transportador de serotonina (5HTT) y la violencia impulsiva en una muestra forense de 153 hombres. Específicamente, un polimorfismo tipo deleción/ inserción en este gen predijo la conducta violenta impulsiva en esta población. ${ }^{53}$ Otros investigadores también han examinado el fenotipo antisocial-conducta disocial encontrando correlaciones con la forma de baja actividad del 5HTTLPR en mujeres con bulimia, ${ }^{54}$ y en hombres y mujeres en una variedad de escenarios, ${ }^{55-58}$ pero Monuteaux et al. (2009) no encontraron asociación entre el 5HTTLPR y los trastornos de conducta en una muestra de individuos con TDAH. ${ }^{59}$

Una vez más, Retz et al. (2008) confirmaron que los portadores de dos alelos largos tenían más síntomas de TDAH en la infancia y la adultez, pero que los sujetos con al menos un alelo corto estaban más sensibilizados para la adversidad en la infancia que aquellos que portaban los dos alelos largos. ${ }^{60}$ Posteriormente, Retz y Rosler (2009) presentaron una interesante revisión selectiva de TDAH, agresión y 5HTTLPR, notando que la literatura científica en general sostenía la idea de que polimorfismos comunes podrían producir efectos diferentes ante circunstancias diferentes; concluyeron que aquellos individuos portadores de dos alelos largos estaban en mayor riesgo para TDAH, pero aquellos con alelos cortos presentaban mayor riesgo para el TDAH y la conducta violenta ante un escenario de estrés ambiental. ${ }^{61}$

Recientes estudios han implicado el gen para el receptor de serotonina 5HTR2A con impulsividad, agresión y conducta antisocial. ${ }^{62-64}$

Un estudio de Dick et al. (2006) encontró que los individuos que tenían un polimorfismo en el gen GRBRA2 asociado con dependencia al alcohol era menos probable que estuvieran casados, en parte porque tenían un riesgo más elevado para el TAP y estaban menos motivados por el deseo de complacer a los demás. ${ }^{65}$ 
Los polimorfismos en el gen MAOA se han asociado con trastornos de personalidad del cluster $\mathrm{B}^{66} \mathrm{y}$ con rasgos antisociales. ${ }^{67}$ El gen MAOA está localizado en el cromosoma X (Xp11.4-p11.3) y codifica para la monoamino oxidasa A, una enzima que degrada los neurotransmisores amínicos, como la dopamina, la noradrenalina y la serotonina. La mutación en este gen da como resultado una deficiencia de monoamino oxidasa, o el síndrome de Brunner, el cual se caracteriza, en parte, por una grave conducta impulsiva. ${ }^{44}$

Basado en los resultados de los estudios cuantitativos de genética que mostraban una interacción gen-ambiente en la conducta antisocial, Caspi et al (2002) ${ }^{68}$ estudiaron la asociación entre el maltrato infantil y un polimorfismo funcional en la región promotora del gen MAOA sobre la conducta antisocial, evaluada por medio de un rango de medidas categóricas y dimensionales utilizando cuestionarios, entrevistas y registros oficiales. Los resultados no mostraron un efecto importante exclusivo del gen, sólo un ligero efecto para el maltrato, pero sí una sustancial y significativa interacción entre el gen y la adversidad. Los niños maltratados que eran portadores para niveles bajos de expresión del gen MAOA, con mayor frecuencia desarrollaron trastornos de conducta y una personalidad antisocial que aquellos niños con un genotipo de alta actividad para MAOA. Foley et al. $(2004)^{69}$ repitieron estos hallazgos y extendieron el análisis inicial al mostrar que la interacción gen-ambiente no podía explicarse por una correlación gen-ambiente; otros estudios han replicado a la vez estos hallazgos del genotipo de baja actividad del MAOA..$^{70-81}$

Otros estudios sugieren que los efectos de los genes no son contribuyentes para la predicción de la conducta disruptiva en aquellos con una historia de maltrato $u$ otros estresores durante el periodo de desarrollo. . $^{8,83}$

Weder et al. (2009) encontraron que la vulnerabilidad era inducida por la MAOA en niveles moderados de trauma ambiental, pero que el genotipo no era contribuyente a la predicción de agresión en niveles extremos de trauma ambiental. ${ }^{84}$ Sjoberg et al. (2008) sugieren que el genotipo MAOA podría interactuar con la testosterona en la predicción del espectro conductual antisocial, aunque no encontraron una asociación directa entre la baja actividad de la variante y la conducta agresiva, ${ }^{85}$ mientras que Beaver et al. (2010) no encontraron efectos directos del genotipo MAOA con las escalas de delincuencia en una muestra de adolescentes; en cambio sí reportaron que el genotipo MAOA parecía interactuar con déficits neuropsicológicos en la predicción de delincuencia. ${ }^{86}$ Buckholtz y Meyer-Lindenberg (2008) revisaron la bibliografía disponible sobre la MAOA en la agresión impulsiva, y propusieron un mecanismo por el cual la variante de baja actividad de la MAOA podría sensibilizar circuitos neuronales pertinentes al estrés en la vida temprana, mientras concluían que la variación de la MAOA VNTR daba razón para sólo una cantidad pequeña de la varianza en el riesgo. ${ }^{87}$
En un meta-análisis de Kim-Cohen et al. (2006), el hallazgo original de Caspi fue replicado. Además, los hallazgos se extendieron para incluir niños (próximos a la fecha del maltrato), y la posibilidad de un hallazgo espurio fue descartado al tomar en cuenta la correlación gen-ambiente. ${ }^{88}$ No todos los estudios han replicado los hallazgos discutidos anteriormente..$^{89-91}$

En relación a la COMT, distintos estudios han implicado al polimorfismo valina/metionina (val/met) en la agresión entre individuos con esquizofrenia, ${ }^{92,93}$ y estudios más recientes sugieren que sujetos homocigotos para el alelo $\mathrm{val} / \mathrm{met}$ tuvieron mayor agresividad..$^{94-97}$

En un estudio de 240 niños con trastorno por déficit de atención con hiperactividad (TDAH), aquellos con la variante valina/metionina en el gen de la catecol O-metiltransferasa (COMT) mostraron más conductas antisociales que aquellos sin la variante. ${ }^{98}$

Wagner et al. (2010) reportaron que mujeres con el polimorfismo val/val, una historia de abuso sexual en la infancia y actual trastorno límite de la personalidad, exhibían menos agresión impulsiva que aquellas que portaban los polimorfismos val/met o met/ met. ${ }^{99}$

Es importante destacar que estos genes no parecen, por sí mismos, causar deterministamente el TAP en el mismo sentido que lo haría el gen mutante de HD, que invariablemente produce la enfermedad de Huntington. Más bien estos genes probablemente interactúen unos con otros de manera que aún resulta poco entendible. Además, probablemente existen muchos otros genes que están involucrados ya sea directa o indirectamente (p.ej., vía interacciones) y que aún no han sido identificados. Pero finalmente estos estudios demuestran que la vulnerabilidad genética y la exposición a la violencia familiar interactúan entre sí para producir el TAP.

\section{UNA PERSPECTIVA EVOLUTIVA}

Aquellos genes que promueven que cierta conducta exista entre los individuos debieron haberse elegido por medio del proceso de selección natural. De manera similar a otros comportamientos que tienen orígenes evolutivos, los comportamientos agresivos también deben ser codificados en los genes, que a la postre serán transmitidos a la descendencia a través de la reproducción sexual. ${ }^{41}$

El TAP puede ser entendido como un bi-producto de la agresión humana normal. Específicamente, la agresividad puede ser definida como la producción de una conducta intencional para causar daño físico o humillación a otra persona que desea evitar dicho daño. Pero la conducta violenta antisocial, además de ser todo lo anterior, se lleva a cabo sin tener cuidado por el bienestar o los derechos de los demás. Por ejemplo, actuar en autodefensa como respuesta a un individuo amenazante podría considerarse como una conducta agresiva, pero no una conducta antisocial. ${ }^{41,100}$ 
Teniendo en consideración la existencia de aparentes subtipos cualitativos de agresión, se puede desarrollar un constructo sensible que hipotetice una dicotomía entre un subtipo impulsivo-reactivo-hostil-afectivo (agresión defensiva) y un subtipo controlado-proactivo-instrumental-depredador (agresión ofensiva). ${ }^{101}$ Aunque la mayoría de los estudios neurobiológicos de la agresión y la violencia desafortunadamente no diferencian entre agresión defensiva y ofensiva, esta distinción puede ser relevante para entender los fundamentos neurobiológicos de la conducta agresiva, más aún si están influidos por circuitos neuronales anatómica y funcionalmente distintos. ${ }^{102}$ Se cree que la propensión para la agresión impulsiva, que es relativamente no planeada y espontanea, pero que culmina en la violencia física, se encuentra asociada con un umbral bajo a la activación de los afectos negativos y con una falla para responder apropiadamente ante las potenciales consecuencias perjudiciales previstas por ese comportamiento. La investigación sociopsicológica subraya la relación entre cognición, emoción y agresión; afectos negativos tales como el miedo y la ansiedad frecuentemente precipitan, acentúan y modulan la conducta agresiva. Así, parece razonable que los circuitos neuronales que afectan los estados emocionales, como el sistema serotoninérgico central, también afectarían la predisposición para las conductas agresivas. ${ }^{103}$

La agresión puede ser considerada como una respuesta adaptativa que puede proporcionar ciertos beneficios, tales como la cooptación o la defensa de recursos, mayores opciones de apareamiento y fidelidad de la pareja, así como un mayor estatus.

Nuestro entendimiento de las conductas antisociales puede edificarse sobre distintos supuestos que se derivan de los modelos de la psicología evolucionista, los cuales serían: 1) La agresión humana es una respuesta normativa y adaptativa que le provee una ventaja selectiva a los individuos (hay que notar que esto no implica que sea moralmente deseable); 2) La limitación de la agresión (p. ej., control del impulso) también es una respuesta normativa $\mathrm{y}$ adaptativa que provee una ventaja selectiva a los individuos; 3) Los instintos de agresión y de control del impulso agresivo responden a estímulos ambientales, o catalizadores, los cuales son procesados cognitivamente con el objeto de seleccionar la respuesta más adaptativa a un determinado estresor ambiental. 4) El cerebro humano ha desarrollado sistemas o "dispositivos" independientes para manejar por separado las unidades encargadas de la agresividad y del control-reducción del impulso agresivo. Estos dispositivos pueden llegar a competir entre sí en algunas ocasiones, particularmente cuando los catalizadores ambientales son ambiguos. ${ }^{104}$

Desde una perspectiva evolutiva, una conducta tan ubicua como la agresión puede ser mejor entendida como una adaptación a las presiones del medio ambiente y que provee una ventaja selectiva a los miembros de la especie. ${ }^{105}$
Aunque la agresión en moderadas cantidades y en proporción a las amenazas del ambiente puede ser benéfica, los altos niveles de agresión pueden claramente ser "demasiado de algo bueno" fenotípicamente hablando. Los altos niveles de agresión pueden situar a un individuo en el riesgo extremo de dañar, o pueden conducirle al rechazo social y privarlo de los beneficios de los grupos sociales, mismos que también contribuyeron a la sobrevivencia de los organismos homínidos individuales. Por lo tanto, no es que un individuo se beneficiará de ser agresivo, sino más bien de saber cuándo serlo y de cuándo controlar tales impulsos. Así como un instinto agresivo puede proveer una ventaja selectiva ante determinadas circunstancias, también un instinto de reducción de la agresividad puede proveer una ventaja selectiva en otras circunstancias. ${ }^{52} \mathrm{El}$ instinto de reducción de la agresividad puede ser sinónimo de lo que frecuentemente se refiere como "control del impulso" o "funciones ejecutivas". Los déficits en porciones del cerebro (p. ej., la corteza de los lóbulos frontales) relacionados con las funciones ejecutivas han demostrado que predicen comportamientos excesivamente agresivos (p. ej., conductas antisociales). ${ }^{106-108}$ Los estudios de neuroimagen documentan que lesiones de la corteza frontal se asocian con agresividad impulsiva, o por lo menos con rasgos agresivos. ${ }^{109}$ Los individuos con TAP tienen $11 \%$ menos materia gris en la corteza prefrontal en comparación con los individuos sin este trastorno. Este fue el caso incluso para individuos sin historia de lesión cerebral. Un estudio encontró resultados similares en individuos violentos, en comparación a los controles no violentos, en la corteza prefrontal, la amígdala y el hipocampo. ${ }^{110}$ Los estudios sobre la corteza prefrontal y la violencia son numerosos, y existen excelentes revisiones sobre el tema. ${ }^{111,112}$

Es importante enfatizar la interacción gen-ambiente, ya que de existir un genotipo antisocial, como cualquier otro genotipo, es poco probable que produzca un patrón conductual estático en todas las situaciones ambientales. Más bien el genotipo produce un rango de conductas con el objeto de permitirle al individuo ajustarse a las diferentes amenazas ambientales. Ambientes con pocas amenazas o tensión son menos probables que produzcan respuestas conductuales antisociales, al contrario de lo que harían ambientes con altas amenazas o tensión. Comprender cuáles situaciones ambientales son más probables que produzcan conductas antisociales en individuos con un genotipo de alto riesgo para la personalidad antisocial podría proveer métodos prometedores para la prevención y la intervención dirigidas a incrementar el rango conductual de los individuos antisociales proveyéndoles de opciones conductuales no agresivas. Desde una perspectiva evolutiva, la forma en la cual interactúan los genes y el ambiente hará al individuo más flexible para hacer frente a una serie de posibles amenazas ambientales. Un organismo conductualmente flexible es inherentemente más adaptativo que uno conductualmente rígido. ${ }^{41}$ 
Como se ha indicado a lo largo del presente texto, la presencia de un significativo componente genético en el TAP sugiere el origen evolutivo de estas conductas. Entender las influencias genéticas de la conducta e identificar los factores genéticos de riesgo en los individuos, puede resultar en tratamientos que teóricamente podrían estar dirigidos temprana y preventivamente hacia aquellos individuos que tengan semejantes factores. Comprensiblemente, la discusión de esas posibilidades incluye considerables preocupaciones éticas. ${ }^{113}$ Esto no presupone que tales técnicas no puedan probar en el futuro su utilidad, sino que deben tomarse con gran cuidado para asegurar que cualquier intervención conductual o médica dirigida a prevenir la violencia se realice sólo bajo estrictas guías y parámetros éticos. ${ }^{41}$

\section{REFERENCIAS}

1. Trastornos de la personalidad. En: López J, Valdés M (eds). DSM-IVTR. Breviario. Criterios Diagnósticos. Barcelona: Masson; 2002.

2. Oldham JM, Skodol AE. Charting the future of axis II. J Personal Disord 2000;14:17-29.

3. Widiger TA, Samuel DB. Diagnostic categories or dimensions? A question for the Diagnostic and Statistical Manual of Mental Disorders-Fifth Edition. J Abnorm Psychol 2005;114:494-504.

4. Widiger TA, Trull TJ. Plate tectonics in the classification of personality disorder-shifting to a dimensional model. Am Psychologist 2007;62:71-83.

5. Krueger RF, Skodol AE, Livesley WJ et al. Synthesizing dimensional and categorical approaches to personality disorders: refining the reseacrh agenda for DSM-V Axis II. Int J Methods Psych Res 2007;16:S65-S73.

6. Robins LN. Deviant children grown up: A sociological and psyhiatric Study of Sociopathic Personality. Baltimore: Williams \& Wilkins; 1966.

7. Langbehn, DR, Cadoret RJ. The adult antisocial syndrome with and without antecedent conduct disorder: Comparisons from an adoption study. Comprehensive Psychiatry 2001;42(4):272-282.

8. Barker ED, Maughan B. Differentiating early-onset persistent versus childhood-limited conduct problem youth. American J Psychiatry 2009;166(8):900-908.

9. Diamantopoulou S, Verhulst FC, Van der Ende J. Testing developmental pathways to antisocial personality problems. J Abnormal Child Psychology 2010;38(1):91-103.

10. Earls F, Mezzacappa E. Conduct and oppositional disorders. En: Rutter M, Taylor E (eds). Child and adolescent psychiatry. Cuarta edición. Oxford: Blackwell; 2003; pp. 419-436.

11. Weinberg W, Harper C, Brumback R. Substance use and abuse: Epidemiology, pharmaco- logical considerations, identification and suggestions towards management. En: Rutter M, Taylor E (eds.). Child and adolescent psychiatry. Oxford: Wiley; 2003; pp. 438-454.

12. Torgersen S. Prevalence, sociodemographics, and functional impairment. En: Oldham J, Skodol A, Bender D (eds.). Essentials of personality disorders. Arlington: American Psychiatric Publishing, Inc.; 2009.

13. Oldham J. Personality disorders. Recent history and the DSM System. En: Oldham J, Skodol A, Bender D (eds.). Essentials of personality disorders. Arlington: American Psychiatric Publishing, Inc.; 2009.

14. Torgersen S, Kringlen E, Cramer V. The prevalence of personality disorders in a community simple. Arch Gen Psychiatry 2001;58:590-596.

15. Zimmerman M, Coryell W. DSM-III personality disorder diagnoses in a nonpatient simple: demographic correlates and comorbidity. Arch Gen Psychiatry 1989;46:682-689.

16. Zimmerman M, Coryell WH. Diagnosing personality disorders in the community: a comparison of self-report and interview measures. Arch Gen Psychiatry 1990;47:527-531.
17. Grant BF, Hasin DS, Stinson FR et al. Prevalences, correlates, and disability of personality disorders in the United States: Results from the National Epidemiologic Survey on Alcohol and Related Conditions. J Clin Psychiatry 2004;65:948-958.

18. Coid J, Yang M, Tyrer P et al. Prevalences and correlates of personality disorder. Br J Psychiatry 2006;423-431.

19. Secretaría de Seguridad Pública [SSP]. Consejo de Menores. Sitio Internet http://www.ssp.gob.mx/application?pageid=cmenores_sub_ $2 \&$ docId $=838$.

20. Samuels J, Eaton WW, Bienvenu OJ III et al. Prevalences and correlates of personality disorders in a community simple. Br J Psychiatry 2002;180:536-542.

21. Consejo de Menores [CM]: Memoria 1996-1999. México: Secretaría de Gobernación; 2000.

22. Consejo de Menores [CM]: Memoria 1996-mayo 1997. México: Secretaría de Gobernación; 1997.

23. Castro ME, García G, Rojas E et al. Conducta antisocial y uso de drogas en una muestra nacional de estudiantes mexicanos. Salud Pública Mex 1988;30(2):216-226.

24. Catro ME, Rojas E, De La Serna J. Estudio epidemiológico sobre el uso de drogas y problemas asociados entre la población estudiantil que asiste a los planteles de Bachilleres. Salud Mental 1988;11(1):35-47.

25. Juarez F, Medina-Mora ME, Berenzon S et al. Antisocial behavior: its relation to selected sociodemographic variables and alcohol and drug use among mexican students. Subst Use Misuse 1998;33(7):1437-1459.

26. Juarez F, Villatoro J, Fleiz C et al. Conducta antisocial, ambiente familiar e interpersonal en estudiantes adolescentes del Distrito Federal. En: AMEPSO. La psicología social en México. México: AMEPS; 2002.

27. Juárez F, Villatoro J, Gutiérrez $\mathrm{M}$ et al. Tendencias de la conducta antisocial en estudiantes del Distrito Federal: mediciones 19997-2003. Salud Mental 2005;28(3):60-68.

28. Bouchard TJ, Loehlin JC. Genes, evolution, and personality. Behav Genet 2001;18:379-393.

29. Ando J, Suzuki A, Yamagata S et al. Genetic and enviromental structure of Cloninger's temperament and carácter dimensions. J Personal Disord 2004;18:379-393.

30. Livesley WJ, Jang KL, Vernon PA. Phenotypic and genetic structure of traits delineating personality disorder. Arch Gen Psychiatry 1998;55:941-948.

31. White CN, Gunderson JG, Zanarini MC et al. Family studies of borderline personality disorder: A review. Harv Rev Psychiatry 2003;11:8-19.

32. Trastornos de la personalidad. En: Sadock B, Sadock V (eds). Kaplan \& Sadock. Sinopsis de psiquiatría. Barcelona: Lippincont Williams \& Wilkins; 2009.

33. Cadoret RJ, Yates WR, Troughton E et al. Genetic-environmental interaction in the génesis of agressivity and conduct disorders. Arch Gen Psychiatry 1995;52:916-924.

34. Torgersen S, Czajkowski N, Jacobson K et al. Dimensional representations of DSM-IV cluster B personality disorders in a population-based simple of Norwegian twins: a multi variate study. Psychol Med 2008;38:1617-1625.

35. Rhee SH, Waldman ID. Genetic and environmental influences on antisocial behavior: a meta-analysis of twin and adoption studies. Psychol Bull 2002;128:490-529.

36. Glatt S, Faraone S, Tsuang M. Psychiatric genetics: A primer. En: Smoller J, Sheidley B, Tsuang M(eds.). Psychiatric genetics. Applications in clinical practices. Arlington: American Psychiatric Publishing, Inc.; 2008.

37. Miles D, Carey G. Genetic and environmental architecture on human aggression. J Pers Soc Psychol 1997;72:207-217.

38. Ferguson C. Genetic contributions to antisocial personality and behavior: A meta-analytic review from an evolutionary perspective. J Soc Psychol 2010;150(2):160-180.

39. Kendler KS, Prescott CA, Myers J et al. The structure of genetic and environmental risk factores for common psychiatric and substance use disorders in men and women. Arch Gen Psychiatry 2003;60:929-937. 
40. Krueger RF, hICKS bm, Patrick CJ et al. Etiologic connections among substance dependence, antisocial behavior, and personality: modeling the externalizing spectrum. J Abnorm Psychol 2002;111:411-424.

41. Hicks BM, Krueger RF, Iacono WG et al. Family transmission and heritability of externalizing disorders : a twin-family study. Arch Gen Psychiatry 2004;61:922-928.

42. Lyons MJ, True WR, Eisen SA et al. Differential heritability of adult and juvenile antisocial traits. Arch Gen Psychiatry 1995;52:906-915.

43. Crowe RR. An adoption study of antisocial personality. Arch Gen Psychiatry 1974;31:785-791.

44. Cadoret RJ, Cain CA, Crowe RR. Evidence for gene-environment interaction in the development of adolescent antisocial behavior. Behav Genet 1983;13:301-310.

45. Cadoret RJ, Yates WR, Troughton E et al. Genetic-environmental interaction in the génesis of aggressivity and conduct disorders. Arch Gen Psychiatry 19995;52:916-924.

46. Jaffe SR, Caspi A, Moffitt TE et al. Nature X nurture: genetic vulnerabilities interact with physical maltreatment to promote conduct problems. Dev Psychopathol 2005;17:67-84.

47. Tuvblad C, Grann M, Lichtenstein P. Heritability for adolescent antisocial behavior differs with socioeconomic status: gen-environment interaction. J Child Psychol Psychiatry 2006;47:734-743.

48. Feinberg ME, Button TMM, Neiderhiser JM et al. Parenting and adolescent antisocial behavior and depression -evidence of genotype $x$ parenting environment interaction. Arch Gen Psichiatry 2007;64:457-465.

49. Hicks BM, South SC, Dirango AC et al. Environmental adversity and increasing genetic risk for externalizing disorders. Arch Gen Psychiatry 2009;66:640-648.

50. Cadoret RJ, Langbehn D, Caspers K et al. Associations of the serotonin transporter promoter polymorphism with aggressivity, attention deficit, and conduct disorder in an adoptee population. Compr Psychiatry 2003;44(2):88-101.

51. Lyons-Ruth K, Holmes BM, Sasvari-Szekely M et al. Serotonin transporter polymorphism and borderline or antisocial traits among lowincome Young adults. Psychiatr Neurosci 2007;17:339-343.

52. Jacob CP, Strobel A, Hohenberger K et al. Association between allelic variation of serotonin transporter function and neuroticism in anxious cluster C personality disorders. Am J Psychiatry 2004;161:569-572.

53. Retz W, Retz-Junginger P, Supprian T et al. Association of serotonin transporter promoter gene polymorphism with violence: Relation with personality disorders, impulsivity and childhood ADHD psychopathology. Behav Sci Law 2004;22:415-425.

54. Steiger H, Richardson J, Joober R et al. Dissocial behavior, the 5HTTLPR polymorphism, and maltreatment in women with bulimic syndromes. American J Medical Genetics Part B: Neuropsychiatric Genetics 2008;147(1):128-130.

55. Gerra G, Garofano L, Santoro G et al. Association between low-activity serotonin transporter genotype and heroin dependence: Behavioral and personality correlates. American J Medical Genetics Part B: Neuropsychiatric Genetics 2004;126(1):37-42.

56. Liao DL, Hong CJ, Shih HL et al. Possible association between serotonin transporter promoter region polymorphism and extremely violent crime in Chinese males. Neuropsychobiology 2004;50(4):284-287.

57. Reif A, Rosler M, Freitag CM et al. Nature and nurture predispose to violent behavior: Serotonergic genes and adverse childhood environment. Neuropsychopharmacology 2007;32(11):2375-2383.

58. Sakai JT, Young SE, Stallings MC et al. Case-control and within-family tests for an association between conduct disorder and 5HTTLPR. American J Medical Genetics Part B: Neuropsychiatric Genetics 2006;141(8):825-832.

59. Caspi A, McClay J, Moffitt TE et al. Role of genotype in the cycle of violence in maltreated children. Science 2002;297:851-854.

60. Monuteaux MC, Biederman J, Doyle AE et al. Genetic risk for conduct disorder symptom subtypes in an ADHD sample: Specificity to aggressive symptoms. J Am Acad Child Adolesc Psychiatry 2009;48(7):757-764.
61. Retz W, Freitag CM, Retz-Junginger $P$ et al. A functional serotonin transporter promoter gene polymorphism increases ADHD symptoms in delinquents: Interaction with adverse childhood environment. Psychiatry Res 2008;158(2):123-131.

62. Retz W, Rosler M. The relation of ADHD and violent aggression: What can we learn from epidemiological and genetic studies? International J Law Psychiatry 2009;32(4):235-243.

63. Burt SA, Mikolajewski AJ. Preliminary evidence that specific candidate genes are associated with adolescent-onset antisocial behavior. Aggressive Behavior 2008;34(4):437-445.

64. Mik HM, Ehtesham S, Baldassarra L et al. Serotonin system genes and childhood-onset aggression. Psychiatr Genet 2007;17(1):11.

65. Preuss UW, Koller G, Bondy B et al. Impulsive traits and 5-HT2A receptor promoter polymorphism in alcohol dependents: Possible association but no influence of person- ality disorders. Neuropsychobiology 2001;43(3):186-191.

66. Dick DM, Agrawal A, Shuckit MA et al. Marital status, alcohol dependence, and GRBRA2: evidence for gene-environment correlation and interaction. J Stud Alcohol 2006;67:185-194.

67. Jacob CP, Muller J, Schmidt $M$ et al. Cluster B personality disorders are associated with allelic variation of monoamine oxidase a activity. Neuropsychopharmacology 2005;30:1711-1718.

68. Williams LM, Gatt JM, Kuan SA et al. A polumorphism of the MAOA gene is associated with emotional brain markers and personality traits on an antisocial index. Neuropsychopharmacology 2009;34:1797-1809.

69. Foley DL, Eaves LJ, Wormley B et al. Childhood adversity, monoamine A genetype, and risk for conduct disorder. Arch Gen Psychiatry 2004;61:738-744.

70. Nilsson $\mathrm{K}$, Sjoberg $\mathrm{R}$, Wargelius $\mathrm{H}$ et al. The monoamine oxidase $\mathrm{A}$ (MAO-A) gene, family function and maltreatment as predictors of destructive behaviour during male adolescent alcohol consumption. Addiction 2007;102:389-398.

71. Kim-Cohen J, Caspi A, Taylor et al. MAOA, maltreatment, and geneenvironment interaction preceding children's mental health: new evidence and a meta-analysis. Mol Psychiatry 2006;11:903-913.

72. Contini V, Marques FZ, Garcia CE et al. MAOA-uVNTR polymorphism in a Brazilian sample: Further support for the association with impulsive behaviors and alcohol dependence. American J Medical Genetics Part B: Neuropsychiatric Genetics 2006;141(3):305-308.

73. Ducci F, Enoch MA, Hodgkinson $C$ et al. Interaction between a functional MAOA locus and childhood sexual abuse predicts alcoholism and personality disorder in adult women. Mol Psychiatry 2008;13:334-347.

74. Enoch MA, Steer CD, Newman TK et al. Early life stress, MAOA, and gene-environment interactions predict behavioral disinhibition in children. Genes, Brain, Behavior 2009;9(1):65-74.

75. Huang YY, Cate SP, Battistuzzi C et al. An association between a functional polymorphism in the monoamine oxidase a gene promoter, impulsive traits and early abuse experiences. Neuropsychopharmacology 2004;29(8):1498-1505.

76. Kinnally EL, Huang YY, Haverly $R$ et al. Parental care moderates the influence of MAOA-uVNTR genotype and childhood stressors on trait impulsivity and aggression in adult women. Psychiatr Genet 2009;19(3):126-133.

77. Nilsson KW, Sjoberg RL, Damberg M et al. Role of monoamine oxidase A genotype and psychosocial factors in male adolescent criminal activity. Biol Psychiatry 2006;59(2):121-127.

78. Prom-Wormley EC, Eaves LJ, Foley DL et al. Monoamine oxidase A and childhood adversity as risk factors for conduct disorder in females. Psychol Med 2009;39(4):579-590.

79. Reif A, Rosler M, Freitag CM, et al. Nature and nurture predispose to violent behavior: Serotonergic genes and adverse childhood environment. Neuropsychopharmacology 2007;32(11):2375-2383.

80. Wakschlag LS, Kistner EO, Pine DS et al. Interaction of prenatal exposure to cigarettes and MAOA genotype in pathways to youth antisocial behavior. Mol Psychiatry 2010;15(9):928-937. 
81. Williams LM, Gatt JM, Kuan SA, et al. Polymorphism of the MAOA gene is associated with emotional brain markers and personality traits on an antisocial index. Neuropsychopharmacology 2009;34:1797-1809.

82. Haberstick BC, Lessem JM, Hopfer CJ et al. Monoamine oxidase A (MAOA) and antisocial behaviors in the presence of childhood and adolescent maltreatment. Am J Med Genet Part B: Neuropsychiatric Genetics 2005;135(1):59-64.

83. Young SE, Smolen A, Hewitt JK et al. Interaction between MAO-A genotype and maltreatment in the risk for conduct disorder: Failure to confirm in adolescent patients. Am J of Psychiatry 2006;163(6):1019-1025.

84. Weder N, Yang BZ, Douglas-Palumberi H et al. MAOA genotype, maltreatment, and aggressive behavior: The changing impact of genotype at varying levels of trauma. Biol Psychiatry 2009;65(5):417-424.

85. Sjoberg RL, Ducci F, Barr CS et al. A non-additive interaction of a functional MAO-A VNTR and testosterone predicts antisocial behavior. Neuropsychopharmacology 2008;33(2):425-430.

86. Beaver KM, DeLisi M, Vaughn $M$ et al. Intersection of genes and neuropsychological deficits in the prediction of adolescent delinquency and low self control. International J Offender Comparative Criminology 2010;54(1):22-42.

87. Buckholtz JW, Meyer-Lindenberg A. MAOA and the neurogenetic architecture of human aggression. Trends Neurosci 2008;31(3):120-129.

88. Kim-Cohen J, Caspi A, Taylor A et al. MAOA, maltreatment, and gene-environment interaction predicting children's mental health: new evidence and a meta-analysis. Mol Psyquiatry 2006;11:903-913.

89. Koller G, Bondy B, Preuss UW et al. No association between a polymorphism in the promoter region of the MAOA gene with antisocial personality traits in alcoholics. Alcohol Alcohol 2003;38(1):31-34.

90. Prichard ZM, Jorm AF, Mackinnon A et al. Association analysis of $\mathbf{1 5}$ polymorphisms within 10 candidate genes for antisocial behavioural traits. Psychiatr Genet 2007;17(5):299-303.

91. Huizinga D, Haberstick BC, Smolen A et al. Childhood maltreatment, subsequent antisocial behavior, and the role of monoamine oxidase $\mathrm{A}$ genotype. Biol Psychiatry 2006;60:677-683.

92. Rujescu D, Giegling I, Gietl A et al. A functional single nucleotide polymorphism (V158M) in the COMT gene is associated with aggressive personality traits. Biol Psychiatry 2003;54(1):34-39.

93. Volavka J, Bilder R, Nolan K. Catecholamines and aggression: The role of COMT and MAO polymorphisms. Ann N Y Acad Sci 2004;1036:393-398.

94. Caspi A, Langley K, Milne B et al. A replicated molecular genetic basis for subtyping antisocial behavior in children with attention-deficit/hyperactivity disorder. Arch Gen Psychiatry 2008;65(2):203-210.

95. DeYoung CG, Getchell M, Koposov RA et al. Variation in the catecholO-methyltransferase Val/Met polymorphism associated with conduct disorder and ADHD symptoms, among adolescent male delinquents. Psychiatr Genet 2010;20:20-24.

96. Kulikova MA, Maluchenko NV, Timofeeva MA et al. Effect of functio- nal catechol-O-methyltransferase Val/Met polymorphism on physical aggression. Bulletin Experimental Biology Medicine 2008;145(1):62-64.

97. Monuteaux MC, Biederman J, Doyle AE et al. Genetic risk for conduct disorder symptom subtypes in an ADHD sample: Specificity to aggressive symptoms. J Am Acad Child Adolesc Psychiatry 2009;48(7):757-764.

98. Thapar A, Langley K, Fowler T et al. Catechol O-Methyltransferase gene variant and birth weight predict early-onset antisocial behavior in children with attention-deficit/hyperactivity disorder. Arch Gen Psychiatry 2005;62:1275-1278.

99. Wagner S, Baskaya O, Anicker NJ et al. The catechol o-methyltransferase (COMT) val/met polymorphism modulates the association of serious life events (SLE) and impulsive aggression in female patients with borderline personality disorder (BPD). Acta Psychiatr Scan 2010;122(2):110-117.

100. Baron R, Richardson D. Human aggression. New York: Plenum Press; 1994.

101. Vitiello B, Stoff DM. Subtypes of aggression and their relevance to child psychiatry. J Am Acad Child Adolesc Psychiatry 1997;36:307-315.

102. Lesch K-P, Merschdorf U. Impulsivity, aggression, and serotonin: a molecular psychobiological perspective. Behav Sci Law 2000;18:581-604.

103. Reif A, Rösler M, Freitag $C$ et al. Nature and nurture predisponse to violent behavior: Serotonergic genes and adverse childhood environment. Neuropsychopharmacology 2007;32:2375-2383.

104. Buss D, Shackelford T. Human aggression in evolutionary psychological perspective. Clin Psychol Rev 1997;17:605-619.

105. Sagan C, Druyan A. Shadows of forgotten ancestors: A search for who we are. New York, NY: Random House; 1992.

106. Mercer K, Selby M. The effects of psychopathy, violence and drug use on neuropsychological functioning. American J Forensic Psychology 2005;23:65-86.

107. Donovan W, Ferraro R. Frontal lobe deficits in domestic violence offenders. Genet Soc Gen Psychol Monogr 1999;125:71-102.

108. Soderstrom H, Hultin L, Tullberg M et al. Reduced frontotemporal perfusion in psychopathic personality. Psychiatry research: Neuroimaging 2002;114:81-94.

109. Leon-Carrion J, Chacartegui-Ramos F. Brain injuries and violent crime. En: Ferguson C (ed.). Violent crime: Clinical and social implications. Thousand Oaks: Sage;

110. Critchley HD, Simmons A, Daly EM et al. Prefrontal and medial temporal correlates of repetitive violence to self and others. Biol Psychiatry 2000;47(10):928-34.

111. Davidson R, Putnam K, Larson C. Dysfunction in the neural circuitry of emotion regulation - a possible prelude to violence. Science 2000;289:591-594.

112. Hare R. Without conscience. New York, NY: Guilford Press; 1993.

113. Williams J. Violence, genes and prejudice. Discover Magazine 1994. Retrieved January 14, 2007, from http://discovermagazine.com/1994/ nov/violencegenesand 446 .

Artículo sin conflicto de intereses 